\section{O4 (continued)}

Hill; Shu Wen Ng, PhD, University of North Carolina at Chapel Hill; Alice Ammerman, DrPH, University of North Carolina at Chapel Hill

Objective: To assess how authentic assignments and class activities impact students' confidence for engaging in policy-related activities.

Use of Theory or Research: Authentic assignments allow students to apply skills they learn in class to real-world situations. Research in social work and nursing has shown students in policy courses with authentic assignments and class activities have improved their knowledge, motivation, confidence, and skills for engaging in policy-related activities.

Target Audience: The target audience was students in a 2-section nutrition policy course offered at the University of North Carolina at Chapel Hill in the fall of 2020. The course is required for Master of Public Health students in the Nutrition and Nutrition/Registered Dietitian concentrations and offered as an elective to others.

Course/Curriculum Description: The online course covered a variety of nutrition policy-related topics, through pre-recorded lectures and a weekly live session. Throughout the course, students completed a variety of authentic assignments and class activities such as a group policy brief project, letter to a legislator, and an elevator pitch on a nutrition policy issue.

Evaluation Methods: Students completed an online pre- and post-survey. Confidence for engaging in policyrelated activities was assessed from a list of 11 policyrelated class activities (ie, writing an effective letter to a legislator, developing a policy drop sheet). We asked students to rate their confidence regarding engaging in these types of policy activities on a scale of 1 (not at all confident) to 5 (very confident). Statistical significance for changes in confidence from pre- to post-survey was assessed using Wilcoxon signed rank tests.

Results: A total of 35 students completed both the pre- and post-surveys. There was a statistically significant increase in confidence for engaging in all activities assessed $(P<0.001)$.

Conclusion: The use of authentic assignments in a nutrition policy course increased students' confidence for engaging in nutrition policy-related activities and is helpful for preparing students to participate in the nutrition policy process.

Funding: None.

\section{Chronic Disease \& Wellness}

\section{Seeing the "Bigger Picture": Impact of an Arts-Focused Type II Diabetes Education Program}

Stephanie Machado,DPH, MPH, ssmachado@csuchico.edu, California State University, Chico, 400 West First St, Chico, CA, 95929; Dean Schillinger, MD, University of California, San Francisco; Lizette Avina, BS, University of California, Berkeley; Ryane Daniels, MPH, University of California, San Francisco; Gabriel Cortez, Youth Speaks; Hannah Thompson, PhD, MPH, University of California, Berkeley
Background: Traditional health education efforts rarely align with youth social justice values. The Bigger Picture (TBP), a public health literacy campaign that uses spoken word art to promote health, leverages a social justice approach to activate youth around the social determinants of type 2 diabetes (T2D).

Objective: We examine the impact of TBP on healthrelated mindsets and expectations; sense of belonging; and civic engagement among youth in urban, low-income high schools.

Study Design, Setting, Participants: In this clusterrandomized study students from 3 high schools received a T2D-related arts-based program and students from $3 \mathrm{com}$ parison schools received a non-health related arts-based program. Both programs were delivered in classrooms and extracurricular clubs over the course of 3 semesters (with the final semester modified due to coronavirus-related school closures).

Measurable Outcome/Analysis: We conducted interviews with adults ( $\mathrm{n}=32 ; 27$ unique participants) and youth $(\mathrm{n}=15)$ from both intervention and comparison schools who participated in programming. In addition, we performed a content analysis of student poems $(n=10)$ related to T2D in intervention schools.

Results: Students in both intervention and comparison schools listed factors like genetics, sugar consumption, diet, income, and neighborhood access to nutrition and physical activity as contributors to T2D. In more cases, however, students in intervention schools were able to draw connections between race/ ethnicity and poor access to basic health-promoting resources. Students in both programs had a high sense of belonging at school and confidence in civic engagement. One instance of T2D-related civic engagement was reported in intervention schools. Content analysis of TBP students' poems revealed youth's detailed understanding the role that government and corporate influence, access, individual behavior, and sociocultural factors play in developing T2D.

Conclusion: Future studies might explore program scalability, and how the integration of civic engagement opportunities into TBP curriculum might impact student's capacity to actively participate in creating social positive change around the social determinants of T2D.

Funding: Robert Wood Johnson Foundation, San Francisco Unified School District and the San Francisco Soda Tax.

\section{Depressive Symptoms, not Food Insecurity, Increased During COVID-19 Pandemic in At-Risk Group with Metabolic Syndrome}

Barbara Lohse,PhD, RDN, balihst@rit.edu, Rochester Institute of Technology, 180 Lomb Memorial Dr 78-A622, Rochester, NY, 14623; Lisa Bailey-Davis, EdD, RDN, Geisinger Health Plan; Betty Drees, MD, FACP, University of Missouri School of Medicine (UMKC); Kevin Masters, $P h D$, University of Colorado Denver; Elizabeth H Ruder, PhD, MPH, RDN, Rochester Institute of Technology; Nicole Trabold, PhD, LMSW, Rochester Institute of Technology 


\section{O6 (continued)}

Background: Metabolic syndrome (MetS) is a risk factor for COVID-19 morbidity and mortality, thus pandemicdriven policies may more strongly influence the emotional responses of those with MetS.

Objective: Examine emotional, mental health, and food security impact of COVID-19 pandemic on persons with metabolic syndrome

Study Design, Setting, Participants: Baseline, prepandemic biomedical measures and online survey of persons enrolled, with minimal or no participation in a 2-year lifestyle intervention trial to reverse metabolic syndrome; repeated online survey during COVID-19 pandemic.

Measurable Outcome/Analysis: Change in depressive symptoms (Patient Health Questionnaire-8 score), perceived stress (Cohen Stress Scale), food security (USDA 10-item Food Security Screener); baseline eating competence (EC; Satter Eating Competence Inventory), health (SF-36), socioeconomic and demographic items. Change in normal and transformed variables was analyzed with paired $t$-tests, multiple regression, repeated measures ANOVA.

Results: Participants ( $\mathrm{n}=132$; MetS diagnosis) were mostly female (67\%), White (70\%), middle-aged (mean $57.0 \pm 11.0$ y) well-educated (62\% college/post-graduate), with a median income of $\$ 86,000$. Perceived stress was significantly higher at baseline than follow-up $(18.5 \pm 6.4$ vs 14.9 $\pm 7.2 ; P<0.001)$ even when controlling for confounders (eg, EC, vitality). Food security increased from $83 \%$ at baseline to $90 \%$ at follow-up $(P<0.001)$. Migration to or continued food insecurity $(n=13)$ tended to be associated with being non-White $(P=0.05)$. Depressive symptoms increased from baseline $(2.1 \pm 2.3$ vs $3.5 \pm 3.4 ; P<0.001)$ even when controlling for EC or gender. Depression, which was a study exclusion criterion, was noted at follow-up $(n=10)$. Movement to depression was not associated with socioeconomic demographics, but was related to lower baseline vitality $(44.4 \pm 20.7$ vs $60.3 \pm 18.9 ; P=0.012)$, mental health $(71.0$ \pm 14.3 vs $82 \pm 10.4 ; P=0.002)$ and higher follow-up perceived stress $(22.6 \pm 7.7$ vs $14.2 \pm 6.8 ; P<0.001)$.

Conclusion: A high-risk sample for COVID-19, which was highly educated, did not experience increased stress or food insecurity, but demonstrated increased depressive symptoms after the onset of COVID-19 pandemic, with some baseline susceptibility noted.

Funding: William G. McGowan Charitable Fund.

\section{Eating Competence Relationship to Biopsychosocial Characteristics in Metabolic Syndrome Replicates the General Population}

BarbaraLohse,PhD, RDN, balihst@rit.edu, Rochester Institute of Technology, 180 Lomb Memorial Dr 78-A622, Rochester, NY, 14623; Lisa Bailey-Davis, EdD, RDN, Geisinger Health Plan; Betty Drees, MD, FACP, University of Missouri School of Medicine (UMKC); Kevin S Masters, PhD, University of Colorado Denver; Elizabeth H Ruder, $P h D, M P H, R D N$, Rochester Institute of Technology; Nicole Trabold, PhD, LMSW, Rochester Institute of Technology
Background: Eating Competence (EC) is associated with positive emotional, physical, and psychological features in healthy people, but the Satter EC framework (ecSatter) has not been examined in persons with metabolic syndrome (MetS).

Objective: Identify how ecSatter relates to socio-demographic, physical, and psychological measures in persons with MetS.

Study Design, Setting, Participants: Baseline biomedical measures and online survey of persons with MetS enrolled in a 2-year efficacy trial of a lifestyle intervention. Rigorous inclusion criteria addressed motivation, cognitive abilities, and comorbidity exclusion.

Measurable Outcome/Analysis: Patient Health Questionnaire-8, Cohen perceived stress scale, USDA 10-item Food Security Screener (FS); Satter Eating Competence Inventory (ecSI 2.0), SF-36 vitality and mental health scales, socioeconomic and demographic items. Pearson r, independent $t$-tests, and chi-square compared ecSI 2.0 scores and EC categories with survey findings and biomedical measures.

Results: Participants $(\mathrm{n}=132)$ were mostly female $(67 \%)$, White $(70 \%)$, middle-aged (mean $57.0 \pm 11.0$ y), well-educated (62\% college/post-graduate), with a median income of $\$ 86,000$. ecSI 2.0 score ranged from 10 to 47 with mean of $30.7 \pm 7.2$. Sixty-two participants (47\%) were denoted as EC, (ecSI $2.0 \geq 32)$. EC persons were older $(59.2 \pm 10.5$ vs $55.1 \pm 11.1 \mathrm{y} ; P=0.03)$, had a lower BMI (35.4 \pm 5.4 vs 38.2 $\pm 8.9 ; P=0.037)$, greater vitality $(63.6 \pm 18.0$ vs $55.0 \pm$ $19.8 ; P=0.01)$ and mental health $(84.2 \pm 8.7$ vs $78.4 \pm 12.3$; $P=0.002)$, less perceived stress $(17.0 \pm 5.8$ vs $19.9 \pm 6.6 ; P=$ $0.007)$ and depressive symptoms ( $1.6 \pm 2.0$ vs $2.5 \pm 2.5 ; P=$ 0.035). FS was high for $92 \%$ of EC and for $74 \%$ of non-EC. Of the 23 with low/very low FS, 22\% were EC and 78\% not EC $(P=0.008)$.

Conclusion: The relationships of ecSatter with biomedical and psychosocial determinants of health in well persons were affirmed in persons living with MetS, supporting education focused on ecSatter tenets in this high-risk group.

Funding: William G. McGowan Charitable Fund.

\section{Translation of the National Diabetes Prevention Program into Cooperative Extension: Barriers and Facilitators}

HannahWilson, BA, hannah.wilson@uga.edu, University of Georgia, 206 Hoke Smith Annex, 300 Carlton St, Athens, GA, 30602; Darci Bell, BS, RDN, University of Georgia; Alison Berg, PhD, LD, RDN, University of Georgia

Background: Translation of the Center for Disease Control's National Diabetes Prevention Program (DPP) from clinics to communities can reduce diabetes burden in the U.S. if more organizations adopt and maintain the intervention. Cooperative Extension offers a potentially powerful context for DPP implementation, with a capacity to reach large and diverse populations. There is a critical 